\title{
Victoria Chick
}

\section{Über Geld und Geldtheorien}

Auf den folgenden Seiten werde ich einen kurzen Streifzug durch jene Teile der geldtheoretischen Literatur des 20. Jahrhunderts unternehmen, deren kritische Betrachtung deutlich macht, wie eine sozialwissenschaftlich gehaltvolle Theorie des Geldes und der Geldwirtschaft beschaffen sein sollte. Ausgehend von einer Kritik der methodischen Grundlagen der allgemeinen Gleichgewichtstheorie werde ich zeigen, daß eine Theorie des Geldes nur im Rahmen solcher Theorien sinnvoll entwickelt werden kann, die offen für historische Prozesse und die evolutionäre Entwicklung von Institutionen sind.

\section{Geld in der Theorie des Allgemeinen Gleichgewichts}

Obwohl der englische Ökonom Frank Hahn, einer der bekanntesten Vertreter der neoklassischen Theorie, feststellt, daß es in der allgemeinen Gleichgewichtstheorie eigentlich keinen richtigen Platz für Geld gibt (Hahn 1982), weist die Theorie von Walras, einem der Begründer der neoklassischen Schule, dennoch eine bestimmte Art des Geldes auf. Spätestens seit den 70er Jahren haben sich die Postkeynesianer darüber beschwert, daß dieses walrasianische Geld ein Gut wie jedes andere sei: alle Güter seien gegeneinander austauschbar und nur formal, durch eine Indexzahl, voneinander unterschieden. In dieser Situation könne ein beliebiges Gut willkürlich zum allgemeinen Wertstandard gewählt werden - zum numéraire. Im Gegensatz dazu versuchte man in den alten Geldtheorien zumindest noch, solche natürlichen Eigenschaften zu suchen, welche bestimmte Güter dazu prädestinieren, als Geld zu dienen. Die Edelmetalle übernahmen demnach die Geldfunktionen, weil sie über solche Merkmale wie die Haltbarkeit, Transportierbarkeit und Teilbarkeit verfügten. In diesen Theorien suchte man also noch nach Gründen dafür, daß die Geldeigenschaft bestimmten Gütern anhaftet. Es wird darin deutlich, daß Geld nichts Zufälliges ist - im Gegensatz zur allgemeinen Gleichgewichtstheorie.

Zumindest aus einem Umstand wird sofort ersichtlich, warum wirkliches Geld mit dem walrasianischen System nicht vereinbar ist. In der neoklassischen Modellwelt werden alle Güter ihres Gebrauchswerts wegen nachgefragt. 
Daher muß Geld ein Gut wie alle anderen sein - es muß einen Gebrauchswert besitzen. Wenn eine Ware wie Gold oder Zigaretten im alltäglichen Leben als Tauschmittel benutzt werden, dann erhöht diese spezielle Nützlichkeit im Austausch zugleich ihren Wert. In der walrasianischen Welt erhält die Ware, welche als Geld dient, keinen zusätzlichen Wert. Folglich übt das Geld in diesem Modell nur eine seiner Funktionen aus, nämlich die des Wertstandards oder der Recheneinheit, es dient hingegen nicht wirklich als Tauschmittel. Seine Neutralität gegenüber der Produktion, der Einkommensverteilung etc., die es in der walrasianischen Theorie der Preisbestimmung hat, kann daher niemanden überraschen. Genausowenig wie die Neutralität der Geldreform überrascht, die Frankreich im Jahre 1960 durchführte, als es hundert alte Francs in einen neuen Franc umbenannte. Diese Neutralität kann aber nicht umstandslos für solche Theorien beansprucht werden, welche auch die anderen Geldfunktionen erklären wollen.

Werfen wir zum Vergleich einen kurzen Blick auf die Keynessche Theorie, in der Geld keinen Gebrauchswert hat. Keynes stellte sich die Frage, warum irgend eine Person außerhalb einer 'Verwahranstalt für Geistesgestörte' für einen längeren Zeitraum Geld halten sollte, das weder einen eigenen Gebrauchswert aufweist noch Erträge abwirft. Im 17. Kapitel seiner Allgemeinen Theorie gibt er eine Antwort auf diese Frage, in der er darauf hinweist, daß alle Vermögensbestände vier Eigenschaften in einem allerdings sehr unterschiedlichen Grade aufweisen: sie gewähren Erträge, sie erfordern Durchhaltekosten, sie verfügen über eine Liquiditätsprämie und über eine erwartete Aufwertung. Lassen wir die letzte Eigenschaft beiseite. Geld, auch wenn es keinen Gebrauchswert besitzt, zeichnet sich dadurch aus, daß seine Durchhaltekosten gering sind, sein Ertrag gleich Null und seine Liquiditätsprämie sehr hoch ist. Ein Vermögensbestand ist liquide, wenn er kurzfristig und ohne Verlust realisiert, also verkauft werden kann, um beispielsweise seinen Eigentümer in den Stand zu setzen, Zahlungen zu leisten. Unter normalen Bedingungen kann Geld sofort und ohne Verlust realisiert werden, das heißt man kann es gegen etwas anderes austauschen. Geld ist vollständig liquide. Dennoch bedeutet der Begriff der Liquidität mehr als nur die Marktgängigkeit einer Ware, denn jedes Gut kann leicht veräußert werden, wenn nur sein Preis niedrig genug ist. Geld bezieht seine Liquidität aus dem Umstand, daß es in einem weiten Umkreis von Transaktionen ein allgemein akzeptiertes Tauschmittel ist. Es besitzt einen sehr ausgedehnten Markt - der in der Tat so umfassend ist, daß er beinahe alle ökonomischen Austauschprozesse und fast alle Wirtschaftssubjekte umfaßt. Dennoch hat Geld keinen Gebrauchswert. In einer Welt, in der man ein Geld benutzt, das ausschließlich einen Tauschwert aufweist, dürfen die ökonomischen Transaktionen folglich nicht aufhören: Niemand möchte auf einem Sack mit nutzlosem Geld sitzenbleiben, wenn die Musik aufhört (vgl. Hicks 1967). 
Aus dieser Überlegung gewinnen wir einen zweiten Einwand gegen die neoklassische Gleichgewichtstheorie, der zeigt, daß das moderne Geld mit diesem System nicht vereinbar ist. Betrachtet man nämlich, wie die Produktion in das Gleichgewichtsmodell aufgenommen wird, so zeigt sich, daß der prozeßhafte, zeitliche Charakter der Produktion in einen einfachen Austausch umgedeutet wird, in dem sich Arbeitsleistungen und Güter gegenüberstehen (so formuliert es explizit Debreu in der Einleitung zu seiner Theory of Value). Die neoklassische Theorie begründet den Austausch damit, daß die gegebene Erstausstattung der Haushalte - ihre Anfangsausstattung mit Gütern - von dem von ihnen präferierten Güterbündel abweicht. Das Gleichgewicht, das ohnehin nicht mehr als die Lösung eines Gleichungssystems ist, gibt also nur die letztliche Verteilung der Güter auf die Haushalte an. Damit ist die Geschichte zu ihrem Ende gekommen: Hat die Ökonomie diese gewünschte Gütermengenstruktur für jeden Haushalt erreicht, ruhen alle ökonomischen Aktivitäten, bis eine exogene Veränderung zu erneuten Tauschakten zwingt. Dieser exogene Wechsel in den wirtschaftlich relevanten Daten kann in einem neuen Güterbündel bestehen, das gewissermaßen vom Himmel fällt. Mit diesem Modell haben wir gerade jene Welt beschrieben, in der ein 'nutzloses' Geld zurückgewiesen wird, weil jeder befürchtet, es in zukünftigen Tauschakten nicht weitergeben zu können. Das Warengeld wird hingegen angenommen und erhält eine Wertschätzung, gerade weil es auch einen Gebrauchswert besitzt.

Im Gegensatz zu dieser reinen Tauschökonomie des walrasianischen Systems, in der es keine wirkliche Zeit gibt, steht die Produktionsökonomie, deren Aktivitäten in die Zukunft gerichtet sind. Letztere reproduziert sich - so die Marxsche Terminologie -, sie wächst und verändert sich. Ein Produktionsmodell ist daher ein offenes System, das Tauschmodell hingegen beschreibt eine Welt, in der alle wirtschaftlichen Tätigkeiten zum Erliegen kommen, sobald jeder Haushalt das hat, was er haben möchte. Es handelt sich um ein geschlossenes System - eine Ökonomie, in der das wirtschaftliche Handeln in einem finalen Gleichgewicht zur Ruhe kommt. Damit wird auch klar, warum das Konzept von Walras und Debreu, die Produktionsökonomie als Sonderfall einer Tauschökonomie zu beschreiben, die falschen Antworten liefert: geschlossene Systeme kann man als Sonderfälle von offenen auffassen, aber nicht umgekehrt.

In einer Produktionsökonomie, die sich nicht nur reproduzieren kann, sondern in der jedermann sicher ist, daß die Reproduktion gelingt ist, kann auf Geld verzichtet werden, weil der Wert anderer Zirkulationsinstrumente, die zudem einen Zinsertrag abwerfen, im Austausch ebenso sicher ist wie der Tauschwert des Geldes. Damit kommen wir zu einem entscheidenden Punkt: Unsicherheit über die Zukunft bildet einen Wesenszug von Geldwirtschaften; Produktionsökonomien sind ebenfalls durch unsichere, zukünftige Markt- 
konstellationen gekennzeichnet, weil sie sich durch die reale historische Zeit bewegen und nicht durch eine abstrakte, ahistorische Zeit. Den wirtschaftlichen Akteuren ist eben nicht zu jedem Zeitpunkt bekannt, ob sich jedes Unternehmen reproduzieren kann oder wie sich die Reproktion der gesamten Volkswirtschaft darstellt. Obgleich die Vertreter der Keynesschen Theorie dieses Problem schon frühzeitig hervorgehoben hatten, wird es erst in jüngerer Zeit stärker beachtet. In den formalisierten Wirtschaftstheorien geht aber dieser Punkt verloren, wenn man versucht, Unsicherheit in ein kalkulierbares Risiko zu verwandeln.

Schließlich sei noch eine dritte Kritik an der Behandlung des Geldes im Rahmen der allgemeinen Gleichgewichtstheorie angeführt: Geld ist ein soziales Phänomen. Es macht keinen Sinn, über ein Geld ohne Gebrauchswert zu verfügen, wenn es niemanden gibt, dem man es übertragen kann. Die selbstgenügsamen Wirtschaftseinheiten, welche methodologisch die allgemeine Gleichgewichtstheorie kennzeichnen, sind weder mit der Theorie noch mit dem tatsächlichen Gebrauch des Geldes vereinbar.

\section{Die Liquiditätspräferenz}

Nachdem Keynes gezeigt hatte, daß die Liquiditätsprämie die Haltung eines Geldes ohne eigenen Gebrauchswert begründet, ging er zur Untersuchung der Motive über, welche zur Geldhaltung führen: dem Transaktions-, dem Vorsichts- und dem Spekulationsmotiv. Diese Motive sind nur allzu bekannt, weniger bekannt sind aber die Inkonsistenzen, die sowohl hinsichtlich der theoretischen Konstruktion als auch der Messung dieser Motive bestehen (Chick 1981). Die Transaktionsnachfrage kann nur als ein Kassenbestand gefaßt werden, der im Durchschnitt über einen gewissen Zeitraum hinweg besteht, die beiden anderen Motive der Geldnachfrage begründen dagegen eine Geldhaltung, die zu einem bestimmten Zeitpunkt gemessen werden kann. Dahinter verbirgt sich das berühmte Problem von Strom- und Bestandsgrößen (Lloyd 1966; Bushaw/Clower 1957). Im Prinzip ist es möglich, diese Inkonsistenzen der Messung dadurch zu beseitigen, daß alle Arten der Geldhaltung in Stromgrößen verwandelt werden; dies ist der übliche Weg. Es bleibt dennoch die Schwierigkeit, daß weder die spekulative Geldhaltung noch die Vorsichtskasse dauerhaft sind, weil das zu diesen Zwecken verwendete Geld keinen Zinsertrag bringt, aber auch keinem letzten Nutzen dient. Ein solcher Versuch würde jedoch noch ein anderes Problem unterschlagen. Die unterschiedlichen Motive der Geldhaltung sind mit unterschiedlichen Zeithorizonten verbunden: der Spekulant hält das Geld nur solange, wie er eine weitere Senkung der Preise für Wertpapiere erwartet, während derjenige, der Geld aus dem Vorsichtsmotiv hält, einen deutlich längeren Zeitraum in Betrachtung zieht. Diese zeitlichen Planungshorizonte lassen sich jedenfalls nicht mit der 
durchschnittlichen Zeitspanne ausgleichen, für die ein gewisser Geldbetrag als Transaktionskasse verfügbar gehalten wird.

Es ist nun interessant, die verschiedenen Antworten auf dieses Problem miteinander $\mathrm{zu}$ vergleichen. Keynes muß diese Schwierigkeiten zwar wahrgenommen, dann jedoch zugunsten höherer theoretischer Zwecke ignoriert haben. Ihm ging es im wesentlichen um die Erarbeitung einer auf der Liquiditätspräferenz beruhenden Zinstheorie. Allen Formalisten, denen die innere Konsistenz der Argumentation als höchste Würde gilt, die im Feld der wissenschaftlichen Arbeit errungen werden kann, läßt Keynes’ Vorgehen das Blut in den Adern gefrieren. Diesem Purismus kann man aber entgegen halten, daß der Übergang von der Motivation zur Geldhaltung, die notwendiger Weise mikroökonomisch ist, zur Makroökonomie einen Kompromiß unausweichlich macht: Das Kunststück besteht darin, den Kompromiß so zu wählen, daß er sowohl mit der übergreifenden theoretischen Konstruktion als auch mit den empirischen Tatbeständen eingermaßen vereinbar ist.

Denis Robertson quälte sich mit diesen Inkonsistenzen in seinem brillianten Buch Banking Policy and the Price Level (1926), aber er löste sie nicht. Das IS-LM-Diagramm in seiner ursprünglichen Form gibt einen Hinweis, dem zahllose Verfasser von Lehrbüchern anschließend gefolgt sind. Statt mit den Motiven der Geldhaltung zu beginnen, vereinfachte Hicks (1937) die Keynessche algebraische Formulierung der Geldhaltung, indem er die gesamte Liquiditätspräferenz als zusammenhängenden Posten auffaßte, dessen Umfang durch das Volkseinkommen und den Zinssatz bestimmt werde. Damit begann die Umwandlung der Theorie der Liquiditätspräferenz in die Theorie der Geldnachfrage. Letztere impliziert einen Grad an Stabilität und Einheitlichkeit, sogar einen gewissen Gebrauchswert, über den das moderne Geld aber nicht verfügt.

Beinahe gleichzeitig mit seinem berühmten Aufsatz aus dem Jahr 1937 trug Hicks A Suggestion for Simplifying the Theory of Money vor, einen Aufsatz, in dem er vorschlug, das Geld als einen Vermögensbestand - als Aktivum innerhalb eines Portfolios zu behandeln. Dieser Vorschlag ging beinahe zwangsläufig damit einher, die mit dem Transaktionsmotiv verbundene Geldnachfrage aus der Untersuchung auszuschließen; später schlug Hicks diesen Weg tatsächlich ein (Hicks 1967: Kap.1) und argumentierte, daß die Geldhaltung aus dem Transaktionsmotiv nicht freiwillig und durch ein individuelles Kalkül, sondern durch zufällige Umstände und die zeitliche Abstimmung der Transaktionen bestimmt sei. Da der Umfang der Transaktionskasse nicht von den bewußten einzelwirtschaftlichen Entscheidungen abhinge, sollte sie nicht in die Theorie der Geldnachfrage aufgenommen werden.

Die Ökonomen Brainard und Tobin (1963) folgten später dem von Hicks formulierten Vorschlag; sie schlossen nicht nur die Nachfrage nach Transaktionskasse aus ihrem Modell des finanziellen Sektors aus, sondern trennten 
auch die finanziellen Transaktionen der Ökonomie von den Einkommensströmen, vom Konsum und vom Sparen, indem sie die Nachfrage nach jedem Vermögensbestand als Funktion seines Anteils am gesamten Vermögens betrachteten. Es handelt sich dabei also um ein reines Modell der Bestandsgrößen; sobald das Bestandsgleichgewicht gefunden ist, wird es keine weitere Bewegung im ökonomischen System geben, es sei denn, daß eine exogene Größe wechselt. Erneut finden wir hier eine Theorie, in der die komparative Statik benutzt werden kann. Die Anwendbarkeit dieser Methode scheint so wünschenswert zu sein, daß man darüber vollständig vergißt, daß sie in der Tat mit den grundlegenden Eigenschaften des Geldes unvereinbar ist - nämlich mit dem Umstand, daß das Geld einen Tauschwert besitzt, daher in Transaktionen eingesetzt wird, in denen es gegen Gütern getauscht wird. Kommen wir schließlich noch zu Milton Friedman (1956), der die Gegenposition zu Hicks einnimmt. Er beginnt seine Analyse mit einem Portfoliomodell - einer Theorie über die Verwendung von Vermögensbeständen also -, in welches alle Forderungen und alle Formen der Vermögensbestände aufgenommen werden. Nach einigen Argumentationsschritten gelangt Friedman schließlich zu einer Variante der alten Cambridge-Gleichung für die Geldnachfrage, in der nun der Kassenhaltungskoeffizient - der Kehrwert der Umlaufgeschwindigkeit des Geldes - eine Funktion des Zinssatzes ist. Er stellt sodann fest, daß entweder die Zinsrate nur geringfügigen Schwankungen unterliege, oder die Elastizität der Vermögensnachfrage sehr klein sei. Damit hat er das bekommen, was er wollte: Sein Modell stellt eine Verallgemeinerung der Keynesschen Theorie der Liquiditätspräferenz dar, in der Fassung, die sie in der Interpretation durch Hicks gefunden hat, aber schließlich kommt bei all dem doch nur wieder die Quantitätstheorie des Geldes heraus. Friedman entwickelt ein reines Modell der Stromgrößen.

\section{Das Geldangebot}

Keynes betrachtete das Geldangebot in der Allgemeinen Theorie als gegeben. Nicht daß es als starr angenommen würde, es wird einfach nicht erklärt. Die Postkeynesianer mißbilligen diese Entscheidung zwar, aber ich denke, daß ich eine gute Begründung dafür geben kann. Wenn man von einem exogenen Geldangebot - oder von exogenem Geld - spricht, dann verbindet man damit die Ansicht, daß die Zentralbank oder der Staat jederzeit in der Lage ist, das Geldangebot zu kontrollieren und zu steuern. In der Auffassung, daß das Geld vom Staat 'gedruckt' werde, findet sie ihren extremen Ausdruck. Im Gegensatz dazu vertreten einige Postkeynesianer die Theorie des endogenen Geldangebots. Sie widmen nun der Frage eine größere Aufmerksamkeit, wie das Geld durch das Bankensystem geschaffen wird. Im Rahmen dieser Untersuchungen entsteht eine neue Frage, über die Keynes hinwegging: Wenn das 
Geld durch die Bankkredite hervorgebracht wird, welche ökonomischen Aktivitäten werden dann auf diese Weise durch Kredite gefördert?

In einem gewissen Sinne hat sich Keynes allerdings doch mit diesem Problem befaßt und wir können daraus ersehen, in welchen Zusammenhang er das Geldangebot in der Allgemeinen Theorie stellte. Zwar beschäftigte er sich nicht mit den Geschäftsbanken, verglich aber die Wirkungen eines vermehrten Geldangebots, das seinen Ursprung in der Finanzierung öffentlicher Arbeiten durch Kredite der Notenbank an den Staat hat, mit denjenigen Wirkungen, die eine expansive Offenmarktpolitik der Zentralbank auf den Markt ausübt. Im letzteren Fall werden die Händler, die im Auftrag der Zentralbank tätig werden, direkt mit der Liquiditätspräferenz der anderen Marktteilnehmer spielen und das Geldangebot soweit erhöhen, wie es notwendig ist, um die Zinsrate auf ein gewünschtes Niveau zu senken. Im ersten Beispiel, in dem es um die Finanzierung des Staatsdefizits geht, wird zuerst das Einkommen um den gleichen Betrag wie die ursprüngliche Zunahme der Geldmenge steigen. $\mathrm{Da}$ aber die Geldnachfrage aus dem Transaktionsmotiv nicht die gesamte zusätzliche Geldmenge aufnehmen kann, wird ein Teil derselben seinen Weg auf den Wertpapiermarkt finden und die Zinsrate senken. Keynes bemerkt sodann, daß es nützlich sei, den Fall der Offenmarktpolitik als den typischen zu betrachten.

Dieses Beispiel zeigt sehr deutlich, wie sorgfältig Keynes darauf bedacht war, die klassische Theorie des Zinses, derzufolge der Zinssatz durch das Sparen und das Investieren bestimmt wird, durch eine andere Theorie zu ersetzen, in welcher der Zinssatz von der Liquiditätspräferenz abhängt. Dieser wesentliche Streitpunkt sollte in einer, aus seiner Sicht unnötigen Beschäftigung mit Detailfragen nicht verloren gehen. Seine Entscheidung, das Geldangebot ohne weitere Ausführungen einfach als gegeben anzunehmen, kann also einer der 'notwendigen Kompromisse' gewesen sein, die einzugehen man gezwungen ist. Man vergleiche nun diesen Keynesschen Ansatz mit demjenigen Friedmans: Um sich vollständig auf das Problem konzentrieren zu können, welche Konsequenzen mit einer Ausdehnung der Geldmenge verbunden sind, die Methoden der Geldentstehung aber beiseite zu lassen, führt er die Voraussetzung ein, daß das Geld von einem Hubschrauber abgeworfen werde. Friedman verfolgte mit seinem Vorgehen den Zweck, jeweils nur eine einzige Veränderung im ökonomischen System zu untersuchen. Da die Geldmenge sich in der Volkswirtschaft aber niemals verändert, ohne daß auch ein bilanzieller Gegenposten wechselt, kommt Friedmans Vorgehen nicht auf eine strategische Entscheidung zur Darstellung komplexer Zusammenhänge, sondern auf ein Märchen hinaus.

Wenn es sich bei Keynes um einen 'notwendigen Kompromiß' handelte, dann stimmte er jedenfalls gut mit den damaligen Marktverhältnissen überein. Die Bank von England konnte nicht nur machtvoll im Wertpapiermarkt 
intervenieren, sondern ihre Bilanzsumme blieb auch vergleichsweise stabil, nachdem die scharfe Rezession der Jahre 1920-1921 überwunden war. Heute liegen die Dinge etwas anders, weil die Wertpapierbestände, die von den Geschäftsbanken und anderen institutionellen Anlegern gehalten werden, im Vergleich mit denen der Zentralbank stark angestiegen sind. Nachdem die durch die Rückkehr zum Goldstandard ausgelöste Krise in die Große Depression übergegangen war, reagierten die Banken mit einer Aufstockung ihrer festverzinslichen Wertpapiere zu Lasten der Kredite, so daß ihr gesamtes Geldangebot nicht gekürzt wurde, sondern relativ stabil blieb.

Damit wird ein weiterer wichtiger, methodischer Gesichtspunkt deutlich: Man kann die Keynessche Annahme eines gegebenen Geldangebots aus theoretischen und aus historischen Gründen verteidigen. Aus letzteren deshalb, weil die Voraussetzungen, die Keynes getroffen hat, durchaus mit den institutionellen Bedingungen und den Marktverhältnissen seiner Zeit übereinstimmten. Vor diesem Hintergrund muß der gegenwärtige Mißbrauch der Idee des exogenen Geldes zur Unterstützung einer neoliberalen Sparpolitik der Regierung und zur Sicherung der geldpolitischen Unabhängigkeit der Zentralbank betrachtet werden. Mit diesem Mißbrauch ist nicht nur eine politische Fehlorientierung verbunden, sondern auch eine falsche methodische Entscheidung. Die Theoriebildung kann in der Ökonomie gerade nicht vollständig frei von allen historischen und institutionellen Bezügen vorangehen. Die ökonomische Theorie ist stets abhängig von historischen Konstellationen, von der besonderen Zeit und dem speziellen Ort.

Wie Hicks einmal bemerkte, ist dieser Zusammenhang an keiner anderen Stelle der Wirtschaftstheorie so deutlich wie beim Geld. Diese enge Beziehung der Geldtheorie zur Geschichte des Geldwesens gewinnt seine besondere Bedeutung auch dadurch, daß drängende geldpolitische Probleme gelöst werden müssen. Hicks hat darin natürlich recht, mein Anliegen an dieser Stelle ist jedoch etwas abstrakter. Betrachtet man allein die Theoriebildung, dann kann eine Theorie der Geldwirtschaft sich nicht in das Reich der allgemeinen Prinzipien flüchten, ohne früher oder später irrelevant zu werden im ungünstigsten Fall, wenn man wirklich an sie glaubt, wird sie gefährlich.

Meine Aufmerksamkeit gilt dem Umstand, daß die Geldtheorie immer an die je besonderen, historischen Konstellationen gebunden ist. Damit kommen wir zum Begriff des offenen ökonomischen Systems. Theorien, die sich mit den wesentlichen Aspekten des Geldes beschäftigen, können keinen der allgemeinen Gleichgewichtstheorie vergleichbaren Endpunkt aufweisen, sie müssen offen sein, wie die Modelle der Produktionsökonomie, und jene Unsicherheit über zukünftige Marktkonstellationen beinhalten, die für die historische Zeit so charakteristisch sind. Im restlichen Teil dieses Artikels werde ich mich auf dieses Problem konzentrieren. 


\section{Offene ökonomische Systeme}

Ein ökonomisches System kann nicht nur zeitlich, sondern auch „räumlich“ offen sein, wobei mit der räumlichen Dimension das ganze Netzwerkt der Institutionen gemeint ist: so ist das Geld mit den Finanzmärkten, der Produktion, dem Sparen der Haushalte - mit tatsächlich jedem Aspekt des Wirtschaftens verbunden. Geld gibt es tatsächlich überall. Wie Keynes bemerkte, muß ein Wirtschaftswissenschaftler seine gesamte Theorie überarbeiten, wenn er sich entscheidet, das Geld als ökonomisches Phänomen wirklich ernst zu nehmen. Häufig - oder sogar in den allermeisten Fällen - wird es indes nur als ein „Schleier" behandelt, der sich über die - in irgendeinem Sinne als 'real' vorgestellte - Ökonomie legt. Obgleich unser ökonomisches Modell für die Allgegenwart des Geldes offen ist, enthält es immer noch zuviel, um alles auf ein Mal zu behandeln. Zwar sind die Methoden zur Bearbeitung dieser Modelle vorhanden, sie werden jedoch eher gering geschätzt. Da gibt es einmal die „babylonische“ Methode, die darin besteht, eine bestimmte Frage unter vielen verschiedenen Gesichtspunkten gleichzeitig zu betrachten (Dow 1985), sowie die entgegengesetzte, auf Alfred Marshall zurückgehende Methode, partielle und zeitliche Schließungen in das Modell einzuführen. Solche Verfahren werden notwendig, um unsere Fragestellungen überhaupt bearbeiten zu können.

Marshalls Methode ist weit verbreitet, wenn das Zeitelement in die ökonomische Theorie integriert wird. Die historische Zeit erstreckt sich nun aber zumindest im Prinzip - vom Urknall bis zum thermodynamischen Ende im allgemeinen Hitzetod. Selbst wenn man die zeitlichen Grenzen enger zieht, sind die Veränderungen vielgestaltig: das Geld hat im Laufe seiner Entwicklung mannigfaltige Formen angenommen, ein Geschäftsbankensystem ist entstanden und hat sein Tätigkeitsfeld immer weiter ausgedehnt, Zentralbanken wurden eingerichtet und ausgebaut. Nur ein Wahnsinniger käme auf den Gedanken, eine Geldtheorie auszuarbeiten, die gleichermaßen für das Mittelalter und die Gegenwart geeignet sein soll, oder eine Produktionstheorie, welche den Feudalismus und den modernen Kapitalismus umschließt. Formuliert man das Problem in dieser Weise, dann wird deutlich, daß eine Theorie über offene ökonomische Systeme nicht ohne Schließungen in zeitlicher Dimension auskommen kann - eine sozialwissenschaftlich fruchtbare Untersuchung muß sich auf typische historische Konstellationen richten.

Marshall teilte die ökonomische Zeit in seinem Konzept der Marktperioden in die lange Frist, die kurze Frist und die tägliche Marktkonstellation - die sehr kurze Frist - ein. Jede dieser Perioden zeichnet sich durch eine bestimmte Gesamtheit von Faktoren aus, die jeweils konstant gehalten wird. In der täglichen Marktperiode werden das Produktionsvolumen und der Kapitalbestand als gegebene und konstante Größen behandelt; in der kurzen Frist 
bleibt der Kapitalbestand konstant, aber das Produktionsvolumen variiert, in der langen Frist kann schließlich auch der Kapitalbestand wechseln. Unter rein methodologischen Gesichtspunkten betrachtet, mag diese Einteilung als willkürlich erscheinen, aber unter ökonomischen Gesichtspunkten erhält sie ihre Relevanz dadurch, daß sie wirkliche, in den Produktions- und Akkumulationsprozessen auftretende Verhältnisse wiedergibt. Die Abgrenzung der Marktperioden hängt also vom jeweiligen Zweck der Untersuchung ab. Es versteht sich von selbst, daß alle Schließungen mit Hilfe der ceteris paribus Klausel im Laufe der Zeit hinfällig werden. Sie dienen lediglich dazu, das ökonomische Denken besser zu organisieren, zunächst nur eine Fragestellung zu bearbeiten, die auf partielle Lösungen führt; anschließend kann man sich mit einem anderen Aspekt des Problems befassen. Wenn die einschränkenden Annahmen zweckmäßig gewählt werden, dann entsprechen sie den wesentlichen Eigenschaften der wirklichen Welt. Ein kurzfristiges Gleichgewicht ist somit immer vorläufig und abhängig von den vereinfachenden ceteris paribus Annahmen, die in jedem einzelnen Fall getroffen wurden. Da diese Schließung stets nur zeitweilig erfolgen kann, bleibt das ökonomische System offen.

Werfen wir nun wieder einen kurzen Blick auf die Theorie des allgemeinen Gleichgewichts: In dieser Welt wird eine Ware irgendwie ausgewählt und zum numéraire erklärt, dennoch sind alle Güter vollständig gegeneinander austauschbar. Wie schauen auf eine vollständig flache, undifferenzierte Landschaft. Wie sollte man darin einen Punkt konstant halten können? Wir können es nicht, und die Theoretiker des allgemeinen Gleichgewichts legen ihn auch nicht fest. Für die heterodoxen Theoretiker stellt sich die Schwierigkeit, daß man in der allgemeinen Gleichgewichtstheorie aus dieser Eigenschaft des Modells eine Stärke macht: Alle relevanten ökonomischen Eigenschaften, die man untersuchen will, werden endogenisiert. Auf diese Weise werden alle Fragen gleichzeitig behandelt. Exogene und endogene Größen sind eindeutig festgelegt. Einem Vertreter der allgemeinen Gleichgewichtstheorie muß Marshalls Vorgehen ausgesprochen seltsam erscheinen: Werden einige Größen für eine gewisse Zeit als Konstante angesehen, so vermuten sie darin eine Unvollkommenheit - aber keine Marktimperfektion, sondern den verderblichen Einfluß eines Theoretikers, der willkürliche oder künstliche Starrheiten in ein ökonomisches Modell schmuggelt. In ihren Augen steigert sich das wissenschaftliche Fehlverhalten zum theoretischen Sündenfall, wenn eine ökonomische Größe im Rahmen einer Gruppe von Voraussetzungen als exogene Konstante, unter anderen Bedingungen aber als endogene Variable betrachtet wird. Ökonomische Kategorien scheinen dann endgültig ihre feste Bedeutung zu verlieren. Dennoch - Marshall und seine Anhänger haben nicht willkürlich argumentiert. Ihrem offenen Modell steht das geschlossene Modell der allgemeinen Gleichgewichtstheorie gegenüber, in dem Geld nur in willkürli- 
cher Weise bestimmt werden kann. Das Ergebnis kennen wir: Zwar ist die Lösung dieses theoretischen Problems elegant, sozialwissenschaftlich aber völlig irrelevant.

\section{Historische Entwicklung und Institutionen}

Die Entstehung und Entwicklung der Institutionen trägt durchaus rätselhafte Züge. Wenn wir wüßten, warum Institutionen notwendig sind, hat Brian Loasby einmal gesagt, dann würden wir sie nicht mehr benötigen. Er meinte damit, daß sie bestimmte Arbeits- und Verfahrensweisen einschließen, die ein undifferenziertes System von atomistischen Akteuren nicht so schnell übernehmen kann, ohne daß aber immer klar wäre, was die Institutionen tatsächlich beitragen. Der Grund dafür liegt zum Teil darin, daß sowohl innerhalb der Institutionen als auch in deren Beziehungen zu den einzelnen Akteuren ein Wissen um Verfahrensweisen wirksam wird, das man als implizites Wissen bezeichnen kann. Es ist nirgendwo schriftlich niedergelegt, es beruht nicht auf formalen Regeln, sondern geht aus langjähriger Übung und aus Konventionen hervor. Jede grundlegende Veränderung macht uns auf die Bedeutung dieses stillschweigenden Wissen aufmerksam.

Die Tatsache, daß Institutionen nicht vollständig durchsichtig sind, bedeutet auch, daß ihre Funktionsweise nicht vollständig auf die Vorteilsmaximierung einzelner Akteure zurückgeführt werden kann. Im Zusammenhang mit unserer Problemstellung spielen Institutionen eine entscheidende Rolle, weil sie zu einer 'Differenzierung der Landschaft' beitragen. Letztere erlaubt eine teilweise Schließung des Modells, die notwendig ist, um auf dem Gebiet der Wirtschaftswissenschaft einen theoretischen Fortschritt zu erreichen. Ähnlich Marshalls Konzept der Marktperioden, das aus empirisch begründeten Ansichten über Produktion und Wachstum entstand, können wir auf die historisch besonderen Funktionsweisen der Institutionen zurückgreifen, um eine ökonomische Theorie der Geldwirtschaft zu entwickeln, die offen im oben beschriebenen Sinne ist. Die Institutionen und die ökonomischen Verhaltensmuster, welche sie erzeugen, entwickeln sich über die Jahre hinweg. Zwei besonders wichtige Punkte sollen in diesem Zusammenhang hervorgehoben werden. Erstens geht es darum, die Perioden so voneinander abzugrenzen, daß man stabile Institutionen mit typischen Funktionsweisen identifizieren kann. Das wird möglich sein, weil es immer Zeitspannen gibt, in denen sich die Entwicklung dieser sozialen Gebilde nur sehr langsam vollzieht. Der zweite wichtige Punkt liegt danach auf der Hand: Man muß auch in der Lage sein zu erkennen, wann ein bestimmtes Institutionensystem mitsamt den ökonomischen Verhaltensweisen, die es hervorgebracht hat, obsolet geworden ist. Dann wird eine neue Theorie notwendig. Damit haben wir aber die Welt der allgemeinen Gleichgewichtstheorie mit ihren evidenten Axiomen, ihren 
logisch konsistenten und eleganten Deduktionen verlassen, statt dessen befinden wir uns in einer Gesellschaft, in der im Widerstreit der Meinungen Urteile über soziale Verhältnisse gefällt werden müssen. Schließlich kommt man an der Einsicht nicht vorbei, daß der Preis für die sozialwissenschaftliche Relevanz der eigenen Theorie darin besteht, daß sie eines Tages hinfällig wird. Keynes beispielsweise war sich vollständig darüber im klaren, daß jede Wirtschaftstheorie an besondere historische Bedingungen gebunden ist. Allerdings hat er es unterlassen, sie in seine eigene Theorie explizit aufzunehmen, so $\mathrm{da}$ es anderen überlassen blieb, sie darin aufzuspüren. Auf diese Weise hat seine Theorie - oder ein schwacher Widerschein derselben - ihre historischen Entstehungsbedingungen für lange Zeit überlebt (Chick 1983).

Das augenfälligste Beispiel dafür ist die Liquiditätspräferenztheorie des Zinses, welche den Zinssatz aus der Disposition über Vermögensbestände erklärt. Die Stärke dieser Theorie gegenüber der lange Zeit dominierenden Theorie der leihbaren Fonds liegt darin, daß sie das große Übergewicht anerkennt, das der Handel mit bereits ausgegebnen Wertpapierbeständen über die Ströme von Borgen und Leihen besitzen, welche für die Theorie der leihbaren Fonds konstitutiv sind. Die Liquiditätspräferenztheorie gilt jedoch nur in einer Konstellation, die dadurch gekennzeichnet ist, daß die Geschäftsbanken Wertpapiergeschäfte in einem großen Umfang betreiben und das Geldangebot in der Hauptsache durch die Offenmarktgeschäfte der Zentralbank bestimmt wird. Unter diesen Umständen wird Geld gegen Wertpapiere getauscht und die Summe beider bleibt annähernd konstant. Sobald aber die Geldversorgung in einem bedeutenden Umfang expandiert, weil die Geschäftsbanken ihre Kreditvergabe ausdehnen oder weil die Verschuldung des Staates stark zugenommen hat, wird sich die Kurve der Liquiditätspräferenz mehr oder weniger stetig verschieben; die Konsequenzen für die Zinsrate sind dann ungewiß. Die Offenmarktgeschäfte sind heute in Großbritannien durch direkte Geschäfte der Zentralbank mit den Geschäftsbanken ersetzt worden, in denen Wertpapierpensionsgeschäfte mit Rückkaufvereinbarung abgeschlossen werden, um die letzteren mit Zentralbankgeld zu versorgen.

Es ist kein Zufall, daß der Aufstieg des Monetarismus in einer Zeit begann, in der die Geldmenge deutlich zunahm. Die Ursache dafür lag bei den Geschäftsbanken, aber nicht bei den Offenmarktgeschäften der Zentralbank. Nur wenige werden den Monetaristen darin widersprechen, daß ein rascher Anstieg des Geldangebots eine erhebliche Wirkung auf das Preisniveau ausübt. In einigen keynesianischen Zirkeln blieb das Phänomen der Stagflation unerklärlich, andere vertraten jedoch die Auffassung, daß die Banken, unterstützt durch die Zentralbank, die damaligen Lohnsteigerungen durch ihre Kreditexpansion mittrugen, bis schließlich die zusätzlich entstandene Geldmenge den Verkauf der Güter zu höheren Preisen unterstützte. Zwar mag die Sprache verschieden gewesen sein, die Vision war indes dieselbe: ein rascher 
Anstieg der Geldmenge kann von den Produktionsprozessen nicht absorbiert werden und schlägt sich in Preisniveausteigerungen nieder (Chick 1984). Gegenwärtig finden wir dieses Phänomen in den USA, insbesondere im Inflationsprozeß an den Kapitalmärkten, der die Liquiditätspräferenz außer Kraft setzt.

\section{Die Evolution der Banken und die Theorie des Bankensystems}

In einem älteren Artikel versuchte ich vor dem Hintergrund der historischen Entwicklung des Bankensystems zu erklären, wie sich die Theorie des Bankensektors herausbildete; besondere Bedeutung kam dabei dem Umstand zu, daß zwischen der Änderung im Geschäftsverhalten der Banken und der theoretischen Reflexion dieser institutionellen Veränderungen eine bisweilen recht lange Zeitspanne liegen kann (Chick 1986). In England begann die Geschichte der Banken damit, daß die Goldschmiede sichere Aufbewahrungsmöglichkeiten für Wertgegenstände anboten. Darauf folgte eine Periode, in der die Banken nur in sehr geringem Umfang Kredite gewährt haben, da sie als bloße Vermittler agierten. Zunehmende Einlagen bildeten hier die Voraussetzung für eine Zunahme im Umfang der gewährten Kredite.

Überspringt man zahlreiche weitere Entwicklungsschritte, so gelangt man zu Verhältnissen, in denen es die Ausdehnung des Bankensystems schließlich ermöglichte, daß die von den Banken ausgegebenen Schuldtitel allgemein als Geld akzeptiert wurden: damit war es möglich, dass die Banken die Einlagen durch ihre Kreditvergabe selbst produzierten, ein Prozeß der lediglich durch die Haltung von Reserven beschränkt wurde. Dieses Entwicklungsstadium war besonders interessant, weil es eine Diskussion darüber entfachte, ob die Banken die Mittel zur Kreditgewährung selbst hervorbrachten oder ob sie gegebene Einlagen einfach nur ausliehen. Allerdings fand diese Debatte zwischen 1920 und 1930 statt - mithin 50 Jahre nach der entscheidenden Änderung im Verhalten der Banken. Nachdem die Geschäftsbanken gelernt hatten, Reserven von der Zentralbank oder voneinander zu borgen, letztere aber noch immer die geldpolitisch entscheidenden Maßnahmen im Markt für Wertpapiere durchführen konnte, wurde die Theorie des Geldschöpfungsmultiplikators glaubwürdig; schließlich anerkannte man die Funktion der Zentralbank als lender of last resort. Damit wurde es den Geschäftsbanken möglich, die Bedingungen ihrer Reservehaltung zu lockern. Durch ein aktives Management ihrer Forderungen, das darauf ausgerichtet ist, dass vor allem solche Einlagen gehalten werden, bei denen die Mindestreserven gering sind, dehnte sich der Spielraum der Geschäftsbanken noch weiter aus. Allerdings brachte dieses Forderungsmanagement auch zusätzliche Kosten mit sich, was die Banken durch zunehmend riskantere Geschäfte im Markt für Derivate auszugleichen versuchten. 
Mit einer solchen Darstellung der Entwicklungsstadien, welche der Bankensektor durchlaufen hat, sollte es möglich sein, die verschiedenen Theorien des Bankensystems auf dessen Geschichte zu beziehen. Jede Entwicklungsperiode muß demnach durch eine eigene Theorie beschrieben werden. In den meisten theoretischen Abhandlungen wird dieser Zusammenhang aber ignoriert. Häufig genug werden die Banken auch heute noch als reine Finanzintermediäre oder - schlimmer - einfach nur als Tresore betrachtet.

\section{Die Kreislauftheorie des Geldes}

Zum Schluß will ich noch einige kritische Anmerkungen zu den Vertretern der sogenannten Kreislauftheorie des Geldes anfügen. Obwohl sie zu den heterodoxen Ökonomen zählen und sogar als Postkeynesianer betrachtet werden, ruft die Methode, Theorie auf historische Konstellationen zu beziehen, ihre Abneigung, wenn nicht sogar ihren Ärger hervor. Die Theorie des Geldkreislaufs beginnt damit, daß alle Wirtschaftseinheiten entsprechend ihrer Funktion zu den drei Sektoren Haushalte, Unternehmen und Banken zusammengefaßt werden; der Staat und die Zentralbank werden als scheinbar überflüssige Institutionen aus der Analyse ausgeschlossen. Die Unternehmen wenden sich an die Banken, um einen Kredit zu erhalten, mit dem sie zum einen die Arbeitskräfte bezahlen und zum anderen den Beginn der Produktionsverfahren finanzieren. Der Kreislauf wird geschlossen, wenn die Unternehmen ihre Produkte an die Haushalte verkauft, letztere diese Ausgaben aus ihren Lohneinkommen bestritten und die Unternehmen schließlich ihre Verbindlichkeiten bei den Banken beglichen haben. Er beginnt von neuem, sobald die Unternehmen wieder Kredite nachfragen, um den Kauf von Vorprodukten zu finanzieren. Im Fahrwasser dieser an sich harmlosen Kreislaufbetrachtung, die nichts Ungewöhnliches bietet - sieht man einmal vom Hauch des Sayschen Theorems ab, der sie umweht, kommen weitere theoretische Sätze daher, die der Kritik bedürfen: Geld ist ein Tauschmittel (was fragwürdig wird, wenn man von allen anderen Geldfunktionen absieht); Geld lagen schon immer Kreditbeziehungen zugrunde; es gibt keinen Multiplikator; es gibt weder Knappheit noch eine Reservebeschränkung für die Banken (Parguez 1996).

In einem kürzlich erschienenen Artikel (Chick 2000) habe ich mich mit dieser Theorie aus einer Perspektive auseinandergesetzt, die man als diejenige des „britischen Postkeynesianismus“ bezeichnen könnte: makroökonomisch ist das wichtigste dabei, daß der Bankkredit seine Bedeutung daraus erhält, daß er zur Finanzierung der Investitionen im Unternehmenssektor benutzt wird und daß das Geld, das auf diese Weise aus den Kreditbeziehungen entstanden ist, die Einkommen entsprechend dem Multiplikator vermehrt. Aus der Sicht dieses britischen Postkeynesianismus sind Unternehmen dauerhafte 
Institutionen: die Umsatzerlöse der letzten Periode finanzieren die laufenden Lohnzahlungen, die Erlöse dieses Jahres die Löhne des nachfolgenden. Die Kreislauftheorie beginnt dagegen bei Null: irgend jemand entscheidet sich, ein Produzent zu werden, obwohl er kein Kapital besitzt; das sind die Unternehmen. Die Banken verfügen auf wundersame Weise gleichzeitig über die Fähigkeit, Geld zu verleihen, und die Reputation, welche die Voraussetzung für die Akzeptanz des von ihnen geschaffenen Kredits ist. Wenn schließlich am Ende des Jahres die Kredite zurückgezahlt werden, verschwindet das Geld aus dem Kreislauf, es bleibt kein Geldbestand übrig, der auf die nächste Periode übertragen wird. Dadurch wird der Multiplikator unwirksam. Dieser Abschluß des Kreislaufs folgt aus dem Umstand, daß jedes Unternehmen seine Produktionsprozesse ausschließlich durch Kredite finanziert hat, so daß die gesamten Umsatzerlöse benötigt werden, um ihre Verbindlichkeiten zu tilgen. Wir sehen somit, daß diese Theorie doch nicht so harmlos ist wie man zunächst meinen konnte. Die ökonomischen Prozesse, die sie behandelt, werden der zeitlichen Dimension vollständig entkleidet: die Unternehmen und die Banken tauchen aus dem Nichts auf, sie haben keine historischen Vorläufer, und wenn der einzelne Kreislauf abgeschlossen ist, bleiben keine ökonomischen Beziehungen übrig, die in die Zukunft weisen. Diese Theorie argumentiert mit einem aus Stromgrößen gebildeten, geschlossenen Modell in der Art, wie Wicksell diese Fragen behandelt hat.

Die Divergenzen, die zwischen meiner Position und den Auffassungen der Theoretiker des Geldkreislaufs bestehen, berühren die Grundüberzeugungen der Theoriebildung. So wie die Anhänger Debreus die allgemeine Gleichgewichtstheorie als die universell gültige betrachten, weil sie außerhalb aller institutionellen Gefüge steht, so sehen die Kreislauftheoretiker gerade in der historischen Beziehungslosigkeit den Vorzug ihrer Modelle. Die erste Einsicht, daß die Bankgeschäfte einmal durch den Zwang zur Reservehaltung beschränkt waren - selbst wenn diese Restriktion heute, darin stimme ich mit ihnen überein, nicht mehr gilt -, betrachte ich als ebenso unverzichtbar wie die zweite, daß das Geld einmal etwas anderes als eine Form des Kredits gewesen ist. Was ich für eine Tugend halte - die Herstellung historischer Zusammenhänge -, gilt ihnen als Laster. So neigen die Theoretiker des Geldkreislaufs stets dazu, überhistorische Wesensbestimmungen zu formulieren: $\mathrm{Da}$ das Wesen des Geldes der Kredit sei, gehört ebenso dazu wie die Idee, daß es keine andere Art der Geldwirtschaft als die auf dem Kredit beruhende geben könne. Die historische Perspektive ist hier eher empirisch und sieht, daß sich Geldwirtschaften entwickeln können; es erscheint ganz plausibel, daß alle Beschränkungen des wirtschaftlichen Handeln letztlich gelockert oder aufgelöst werden, weil Institutionen lernen, Restriktionen zu umgehen. Sie hingegen hegen die Idee, daß ökonomische Aussagen nur auf der Grundlage von Lionel Robbins' ebenso berühmter wie ahistorischer Definition des 
Wirtschaftens zu machen sind, derzufolge wirtschaftliches Handeln in der Wahl zwischen alternativen Zwecken besteht, die mit knappen Mittel zu erreichen sind.

Unglücklicherweise erhält diese Schule gerade in einer Zeit Zulauf, in der die Geldsysteme zahlreicher Länder in eine schwierige und gefährliche Lage geraten sind. Während die Kreislauftheoretiker und die britischen Postkeynesianer, zu denen ich mich zähle, sich über die Frage streiten, ob die Banken die Investitionen oder die Vorleistungen durch ihre Kredite finanzieren, gewähren die Banken tatsächlich in großem Umfang Konsumentenkredite und spekulieren in Derivaten. Solange Kredite gewährt werden, um Produktionsprozesse zu finanzieren, die ihrerseits wieder Einkommen schaffen, besteht die Chance, daß die entstehenden Profite die Schuldner in die Lage versetzen den Schuldendienst gegenüber den Banken zu bedienen. Je weiter sich die Bankkredite jedoch vom Einkommenskreislauf entfernen, um so riskanter und krisenanfälliger werden sie. Dieses Beispiel soll nur demonstrieren, daß die Kreislauftheorie des Geldes nicht nur wegen ihrer geschlossenen, ahistorischen Modelle kritisiert werden muß, sondern gerade auch deshalb, weil sie unsere Aufmerksamkeit von möglichen Instabilitäten des Bankensystems ablenkt.

\section{Schlußfolgerung}

In modernen Wirtschaftssystemen ist Geld allgegenwärtig. Die Vorstellung, Geld sei so etwas wie ein Schleier, der sich über die tatsächlichen ökonomischen Aktivitäten legt, kann nur von jemandem aufrecht erhalten werden, der niemals Zeitung liest. Die Volkswirtschaft ist ein offenes, evolutionäres System, das durch Institutionen geformt wird, und nur innerhalb eines solchen Systems kann Geld sinnvoll untersucht werden. Wie ich oben ausgeführt habe, ist die theoretische Erfassung offener Systeme nur möglich, wenn man Schließungen einführt, die den Gegenstandsbereich zeitlich und seinem Umfang nach einschränken. Dabei hängt die Art dieser partiellen Schließung von der Art der Fragestellung ab, sie versteht sich nicht von selbst, sondern beruht auf einer Entscheidung des Theoretikers. Die Komplexitäten und Widersprüche, in denen man sich beim Studium des Geldes bewegt, sind unausweichlich. Allerdings zeigt die Allgegenwart des Geldes auch, daß das, was man in einer Theorie des Geldes lernen kann, die Ökonomie als Ganze betreffen.

Übersetzung aus dem Englischen von Klaus Schabacker 


\section{Literatur}

Bushaw, D.W.; Clower, R.W. (1957): Introduction to Mathematical Economics, Homewood, Irwin Brainard, W.S.; Tobin, J. (1963): Financial Intermediaries and the Effectiveness of Monetary Control, in: American Economic Review, Papers and Proceedings.

Chick, V. (1981): On the Structure of the Theory of Monetary Policy, in D. Currie, R. Nobay and D. Peel, (eds.), Macroeconomic Analysis: Essays in Macroeconomics and Econometrics, Croom Helm, London, 1981, 178-208.

- (1983): A Question of Relevance: The „General Theory” in Keynes's Time and Ours, South African Journal of Economics, 5, September, 388-406.

- (1984): Monetary increases and their consequences: streams, backwaters and floods, in A. Ingham and A. M. Ulph (eds), Demand, Equilibrium and Trade: Essays in Honour of Ivor F. Pearce, London: Macmillan..

- (1986): The Evolution of the Banking System and the Theory of Saving, Investment and Interest, Economies et societes, Cahiers de l'ISMEA, Serie 'Monnaie et Production', No.3, 1986, $111-126$

- (2000) Money and Effective Demand, in J. Smithin, (ed.), What is Money?, Routledge, pp. 124 138.

Debreu, G. (1959):. Theory of Value, New York, John Wiley and Sons.

Dow, S. C. (1985): Macroeconomic Thought: A Methodological Approach. Oxford: Blackwell. Republished in revised form as The Methodology of Macroeconomic Thought. Aldershot: Elgar, 1996

Friedman, M. (1956): The Quantity Theory of Money: A Restatement, Studies in the Quantity Theory of Money (University of Chicago Press) pp. 3-21.

Hahn, F. (1982): Money and inflation, Oxford: Blackwell.

Hicks, J. R.(1937): Mr Keynes and the 'Classics'. A Suggested Interpretation, in: Econometrica, Vol. 5

- (1967) Critical Essays in Monetary Theory, Oxford: Oxford University Press.

Keynes, J. M. (1936) The General Theory of Employment, Interest, and Money, London: Macmillan.

Lloyd, C. L. (1960): The equivalence of the liquidity preference and loanable funds theories and the new stock-flow analysis, Review of Economic Studies 27: 206-09.

Parguez, A. (1996): A reappraisal of the theory of the monetary circuit, in G. Deleplace and E. J. Nell (eds.), Money in Motion: the Post Keynesian and Circulation Approaches, London: Macmillan.

Robertson, D. H. (1926): Banking Policy and the Price Level (London: P. S. King \& Son). 